\title{
ON THE METRIC REPRESENTATIONS OF AFFINELY CONNECTED SPACES
}

\author{
BY T. Y. THOMAS
}

In a recent paper in the Transactions of this Society,* I defined the general concept of the algebraic characterization in complex differential geometry and proved the non-existence of a simple algebraic characterization of the metric spaces in the class of all complex affinely connected spaces. The following note is intended as an addendum to this paper which is to be consulted for the notations and definitions here used.

Assume that $F_{1}=0, F_{2} \neq 0$, where the $F^{\prime}$ 's represent polynomials in the components of the affine connection and their derivatives to an arbitrary but finite order, is necessary and sufficient for a complex affinely connected space to admit an $n$-dimensional metric representation. Then $B_{\beta \gamma \delta}^{\alpha}=0$ implies $F_{2} \neq 0$. Hence one of the polynomials $P$ of the set $F_{2}$ must involve a non-vanishing constant term $C$ so that we may put $P=Q+C$ with the understanding that each term of the polynomial $Q$ depends on a component $\Gamma$ or its derivative.

Consider the one parameter family of metric spaces $S_{a}$ defined in a neighborhood $U$ of the origin of the complex $n$-dimensional number space. For the affinely connected space $S$ defined as the limit space of $S_{a}$ as $a \rightarrow 0$, we have

where

$$
\begin{array}{ll}
\Gamma_{I I}^{1}=-\frac{1}{2} \theta_{1}, & (I=2, \cdots, n), \\
\Gamma_{1 \alpha}^{1}=\frac{1}{2} \theta_{\alpha}, & (\alpha=1, \cdots, n),
\end{array}
$$

$$
\theta=\log ^{-} \phi, \quad \theta_{\alpha}=\frac{\partial \theta}{\partial x^{\alpha}},
$$

and $\phi$ is an analytic function of the coordinates $x^{1}, \cdots, x^{n}$ in the neighborhood $U$ different from zero at $x^{\alpha}=0$; all other com-

* Algebraic characterizations in complex differential geometry, vol. 38 (1935), pp. 501-514. 
ponents of affine connection of $S$ vanish identically in $U$. Now take $\log \phi=\epsilon X^{1}$, where $\epsilon$ is a positive constant; then $\theta_{1}=\epsilon$ and $\theta_{I}=0$, so that all derivatives of the $\Gamma$ 's vanish. Corresponding to any positive number $\eta<|C|$ we can therefore choose $\epsilon$ so that $|Q|<\eta$ in $U$. Then $P \neq 0$ in $U$ for the space $S$. But for the space $S$ we know that $F_{1}=0$. Hence $F_{1}=0, F_{2} \neq 0$ holds in $U$ for the space $S$ so that by the above hypothesis $S$ admits an $n$-dimensional metric representation. But this is in contradiction with the result in $\$ 7$ of the Transactions paper since

$$
\frac{\partial \theta_{I}}{\partial x^{I}}+\frac{\partial \theta_{1}}{\partial x^{1}}+\frac{1}{2} \theta_{I}{ }^{2}+\frac{1}{2} \theta_{1}^{2}=\frac{1}{2} \epsilon^{2} \neq 0
$$

in $U$. It is evident from $\S 8$ of the Transactions paper that the above considerations extend immediately to the case of metric representations of dimensionality $2, \cdots, n-1$, and this gives us the following result: The $r$-dimensional metric representations of a complex affinely connected space $S$, where $r$ is an integer of the set $2, \cdots, n$, do not admit an algebraic characterization.

The problem of determining whether or not a given complex affinely connected space admits an $r$-dimensional metric representation can be reduced to the consideration of a system of linear homogeneous equations in a set of symmetric unknowns $g_{\alpha \beta}$. If this system admits a solution $g_{\alpha \beta}(x)$ such that the matrix $\left\|g_{\alpha \beta}(x)\right\|$ is of rank $r$ for some value of the coordinates $x^{1}, \cdots, x^{n}$, the $r$-dimensional metric representation will exist (see $\$ 6$ of the Transactions paper). In this sense we have an algebraic test for the existence of the $r$-dimensional metric representations. These facts, namely, the existence of the algebraic test and nonexistence of the algebraic characterization of the $r$-dimensional metric representations of a complex affinely connected space $S$, are interesting and instructive inasmuch as differential geometers have so far failed to embark on a critical discussion of the possible types of conditions for the various properties of spaces that have been treated.

Princeton University 